\title{
Recent patent applications in genomic mapping
}

\begin{tabular}{|c|c|c|c|c|c|}
\hline Patent number & Description & Assignee & Inventor & $\begin{array}{l}\text { Priority } \\
\text { application } \\
\text { date }\end{array}$ & $\begin{array}{c}\text { Publication } \\
\text { date }\end{array}$ \\
\hline US 20120064521 & $\begin{array}{l}\text { A method for detecting DNA hydroxymethylation in a DNA sample com- } \\
\text { prising mammalian genomic DNA involving contacting a DNA sample } \\
\text { comprising a glycosylated hydroxymethylcytosine with a DNA endo- } \\
\text { nuclease to cleave the DNA and detecting at least one DNA sequence } \\
\text { from sample not cleaved by the DNA endonuclease to determine the } \\
\text { presence of hydroxymethylation in the DNA sequence. }\end{array}$ & Jia X, Yen J & Jia X, Yen J & 9/9/2010 & $3 / 15 / 2012$ \\
\hline US 20120053082 & $\begin{array}{l}\text { A method for chromosomal mapping comprising providing a sample of } \\
\text { a nucleic acid and exposing the sample to a physical genomic analysis } \\
\text { composition comprising oligonucleotides of different sequences. }\end{array}$ & $\begin{array}{l}\text { Barrett MT, } \\
\text { Caren MP }\end{array}$ & $\begin{array}{l}\text { Barrett MT, } \\
\text { Caren MP }\end{array}$ & $4 / 7 / 2006$ & $3 / 1 / 2012$ \\
\hline US 20120035354 & $\begin{array}{l}\text { A polynucleotide isolated from Eucalyptus grandis and Pinus radiata, } \\
\text { useful for modifying content, structure and composition of lignin in tar- } \\
\text { get organisms such as plants; useful for wood processing for producing } \\
\text { paper, genome mapping, physical mapping, positional cloning of genes, } \\
\text { and designing oligonucleotide probes and primers. }\end{array}$ & $\begin{array}{l}\text { ArborGen } \\
\text { (Summerville, } \\
\text { SC, USA), } \\
\text { Rubicon Forests } \\
\text { Holdings } \\
\text { (Auckland, NZ) }\end{array}$ & $\begin{array}{l}\text { Bloksberg LN, } \\
\text { Havukkala I }\end{array}$ & 9/11/1996 & 2/9/2012 \\
\hline US 20120030602 & $\begin{array}{l}\text { A kit comprising a computer-readable storage medium comprising } \\
\text { a program for producing a graphical user interface used for viewing } \\
\text { genomic array data produced by array-based comparative genome } \\
\text { hybridization and cytogenetic data for cytogenetic assays. }\end{array}$ & $\begin{array}{l}\text { Barrett MT, } \\
\text { Caren MP }\end{array}$ & $\begin{array}{l}\text { Barrett MT, } \\
\text { Caren MP }\end{array}$ & 6/23/2006 & 2/2/2012 \\
\hline WO 2012008831 & $\begin{array}{l}\text { Ordering of sequence tags from part of a genome by generating clone } \\
\text { aliquots from a genomic clone library, generating sequence tags } \\
\text { and ordering the sequence tags based on the combined presence of } \\
\text { sequence tags in the clone aliquots; useful for generating a physical } \\
\text { map of part of a genome and detecting genomic variation between two } \\
\text { samples. }\end{array}$ & $\begin{array}{l}\text { Keygene } \\
\text { (Wageningen, } \\
\text { NL) }\end{array}$ & $\begin{array}{l}\text { Michiels A, } \\
\text { van Eijk MJT, } \\
\text { van Oeveren AJ }\end{array}$ & $7 / 13 / 2010$ & 1/19/2012 \\
\hline US 20120016595 & $\begin{array}{l}\text { A method of transcript mapping by identifying an occurring segment } \\
\text { from a } 5^{\prime} \text { and } 3^{\prime} \text { terminal tag from a transcript of a gene, and identify- } \\
\text { ing feasible gene location, which has a sequence length not exceeding } \\
\text { a predefined gene length. }\end{array}$ & $\begin{array}{l}\text { Agency for } \\
\text { Science, } \\
\text { Technology } \\
\text { and Research } \\
\text { (Singapore) }\end{array}$ & $\begin{array}{l}\text { Ruan Y, } \\
\text { Sung WKK }\end{array}$ & $9 / 13 / 2004$ & $1 / 19 / 2012$ \\
\hline $\begin{array}{l}\text { WO } 2010099301 \\
\text { US } 20110311506 \\
\text { EP } 2401376\end{array}$ & $\begin{array}{l}\text { A transposon comprising at least one hyperactive piggyBac nucleic } \\
\text { acid sequence or its variant(s), derivative(s) or fragment(s) that retains } \\
\text { transposon activity; useful in gene transfer systems for gene therapy, } \\
\text { insertional mutagenesis, gene tagging and gene discovery, e.g., genome } \\
\text { mapping. }\end{array}$ & $\begin{array}{l}\text { Johns Hopkins } \\
\text { University } \\
\text { (Baltimore) }\end{array}$ & Craig NL & 2/25/2009 & $\begin{array}{c}9 / 2 / 2010 \\
12 / 22 / 2011 \\
1 / 4 / 2012\end{array}$ \\
\hline $\begin{array}{l}\text { WO } 2010091248 \text {, } \\
\text { US } 20100204921 \text {, } \\
\text { EP } 2399214\end{array}$ & $\begin{array}{l}\text { A method of selecting one or more markers associated with a trait of } \\
\text { interest in a species of interest, comprising identifying markers associ- } \\
\text { ated with the trait of interest in a population of the species using a } \\
\text { suitably programmed computer to perform genome-wide association } \\
\text { mapping. }\end{array}$ & $\begin{array}{l}\text { Syngenta } \\
\text { Participations } \\
\text { (Basel) }\end{array}$ & $\begin{array}{l}\text { Byrum J, } \\
\text { Clarke JDV, } \\
\text { Guo Z, } \\
\text { Gutierrez RLA, } \\
\text { Kishore VK, } \\
\text { Li M, Wang D }\end{array}$ & 2/6/2009 & $\begin{array}{l}8 / 12 / 2010 \\
8 / 12 / 2010 \\
12 / 28 / 2011\end{array}$ \\
\hline $\begin{array}{l}\text { WO } 2008112754 \text {, } \\
\text { US } 20080228457\end{array}$ & $\begin{array}{l}\text { A computer-accessible medium including a processing arrangement } \\
\text { that obtains one detectable oligonucleotide probe hybridized to at least } \\
\text { one double-stranded nucleic acid molecule cleaved with one restric- } \\
\text { tion enzyme. The location of the detectable oligonucleotide probe is } \\
\text { detected to generate a genome wide probe map. }\end{array}$ & $\begin{array}{l}\text { New York } \\
\text { University } \\
\text { (New York) }\end{array}$ & $\begin{array}{l}\text { Anantharaman T, } \\
\text { Lim S, Mishra B }\end{array}$ & $3 / 12 / 2007$ & $\begin{array}{l}\text { 9/18/2008, } \\
9 / 18 / 2008\end{array}$ \\
\hline
\end{tabular}

Source: Thomson Scientific Search Service. The status of each application is slightly different from country to country. For further details, contact Thomson Scientific, 1800 Diagonal Road, Suite 250, Alexandria, Virginia 22314, USA. Tel: 1 (800) 337-9368 (http://www.thomson.com/scientific). 